\title{
USE OF COMPUTER EXPERIMENTS TO STUDY THE CURRENT COLLECTED BY CYLINDRICAL LANGMUIR PROBES
}

\author{
Tejero-Del-Caz A. ${ }^{a, *}$, Díaz-Cabrera J. M. ${ }^{b}$, Fernández Palop J. I. ${ }^{a}$, \\ BALlESTEROS J. ${ }^{a}$ \\ a Departamento de Física, Universidad de Córdoba, Campus de Rabanales, E-14071, Córdoba, Spain \\ ${ }^{b}$ Departamento de Ingeniería Eléctrica, Universidad de Córdoba, Campus de Rabanales, E-14071, Córdoba, Spain
}

* f52tecaa@uco.es

\begin{abstract}
A particle-in-cell simulation has been developed to study the behaviour of ions in the surroundings of a negatively biased cylindrical Langmuir probe. Here, we report our findings on the transition between radial and orbital behaviour observed by means of the aforementioned code. The influence of the ion to electron temperature ratio on the transition for different dimensionless probe radius is discussed. Two different behaviours have been found for small and large probe radii.
\end{abstract}

Keywords: computer experiments, particle-in-cell, sheaths, Langmuir probes.

\section{Introduction}

The study of the contact of a plasma with a surface has several applications across a wide variety of technological and scientific areas [1-7]. On the one hand, a number of surface treatment and functionalization techniques rely on this precise knowledge. In particular, the vast majority of the microchip manufacturing industry depends on the contact between plasmas and surfaces. On the other hand, the knowledge about the contact of a plasma with a metallic surface, $i . e$. Langmuir probe, can be used to diagnose different plasma parameters such a temperatures and densities of the different species present in the plasma.

The use of Langmuir probes is widespread because it is one of the few plasma diagnosing techniques that provides local information about the plasma parameters. The diagnose with Langmuir probes is performed by comparing the current collected by the probe when biased with respect to the plasma, $i$. e. the $I-V$ characteristic curve, with the prediction of a certain theoretical model. The qualitative shape of a full $I-V$ characteristic curve can be seen in Figure 1. where three distinguished zones are shown depending on the biasing potential of the probe. Those three zones are usually known as: (a) ion saturation, (b) electron retarding and (c) electron saturation.

The ion saturation zone of the $I-V$ characteristic results of particular interest when it comes to diagnose a plasma. This zone is defined by a probe biasing potential, $\phi_{p}$, that is highly negative with respect to the plasma potential. Under this conditions the probe retards electrons and attracts positive ions, so the current collected is mainly due to ions. As can be seen in Figure 1 the current collected in the ion saturation zone is much smaller than the current collected in the electron zones of the characteristic. For this reason, the current drained from the plasma by the probe is diminished in that zone, and the perturbation introduced by the presence of the probe is minimised.

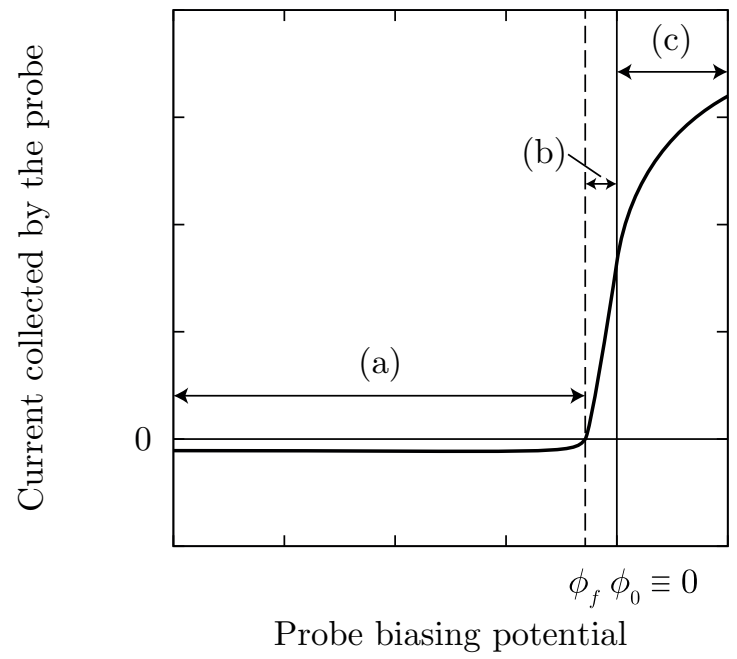

Figure 1. Qualitative description of a current-voltage characteristic curve of a Langmuir probe. The plasma potential, $\phi_{0}=0$, and the floating potential, $\phi_{f}$, divide the characteristic into: (a) ion saturation, (b) electron retarding and (c) electron saturation zones.

Numerous theoretical models 88 14 have been developed over the years in order to predict the ion current collected by a Langmuir probe when negatively biased with respect to the plasma. Particularly controversial are the cases of cylindrical and spherical Langmuir probes (this contribution is centred on the cylindrical case), where there are two main and opposite approaches: radial and orbital theories. While in the former, ions are considered to follow radial trajectories when falling towards the probe, in the latter they move following trajectories that can orbit the probe and go back to the plasma without hitting the surface of the probe, and thus not contributing to the current collected by it. On the one hand, the basic 
orbital theory (OML) was developed by Mott-Smith and Langmuir [8], and then extended by Bernstein and Rabinowitz 9 as well as Laframboise [10, among others. On the other hand, the basic radial theory (ABR) was developed by Allen, Boyd and Reynolds [11 for the case of spherical probes and later on extended by Chen 12 for cylindrical probes. This theory was also extended by the authors [13, 14] to include the positive ion thermal motion.

It is precisely this multiplicity of theories what makes the use of the ion saturation zone controversial for diagnosing purposes, as it is not always clear which theoretical model should be used. Some authors [10] consider that, for the case of cylindrical Langmuir probes, the ABR model is incorrect, arguing that the angular momentum of ions should be described by an isotropic distribution instead of being null, as Chen considered [12. However, depending on the particularities of the experimental setup and the plasma conditions, some experimentalist have found results in agreement with the OML theory [15, 16, while others have found results in agreement with the ABR theory [17, 18. Even more, there are authors who have found results in between both theories [19, and, recently, the authors [20] have experimentally observed a transition between both the ABR and OML theories in a Helium plasma. These results denote a lack of knowledge about a theoretical model that properly describes the behaviour of ions in the surroundings of a cylindrical Langmuir probe immersed in a collisionless plasma when it is being negatively biased with respect to the plasma.

It is our interest to study the role of different parameters in such a transition and, ultimately, to be able to develop a theoretical model that can describe the behaviour of ions in any conditions. In this contribution we report our findings about the influence of the ion to electron temperature ratio, $\beta=T_{i} / T_{e}$, and the dimensionless probe radius, $R_{p}=r_{p} / \lambda_{D}$, on the aforementioned transition. $T_{i}$ and $T_{e}$ being the ion and electron temperature respectively, $r_{p}$ the radius of the probe and $\lambda_{D}$ the Debye length. The results that we are going to show have been obtained by means of computer experiments [21, which results the best technique to study the influence of individual parameters on complex systems such as the one we are dealing with.

\section{Simulation}

In order to study the influence of different parameters on the ion current collected by a probe in the ion saturation zone, a computer experiment has been set up. We have used a particle-mesh (PM) model of the contact of a collisionless plasma with a cylindrical probe. In particular, the developed code is based on a classical electrostatic collisionless particle-in-cell (PIC) algorithm. Because of the symmetry of the problem, the dimensionality of the PIC code is $1 \mathrm{~d} 2 \mathrm{v}$. Obviously, the component of the position considered for every particle is the radial distance from the axis of the probe, while the radial as well as the azimuthal components of the velocity are taken into account.

In Figure 2 the domain of the simulation can be seen. The length of the simulation is set large enough for the influx surface to be located at some point along the presheath, $i$. e. where the quasineutral condition holds. Whereas the width and amplitude of the simulation are adjusted in order to have a reasonable number of particle in the simulation. As the problem presents axial and azimuthal symmetry, it does not matter how large or small the simulation domain is considered, as long as the number of particles is statistically meaningful.

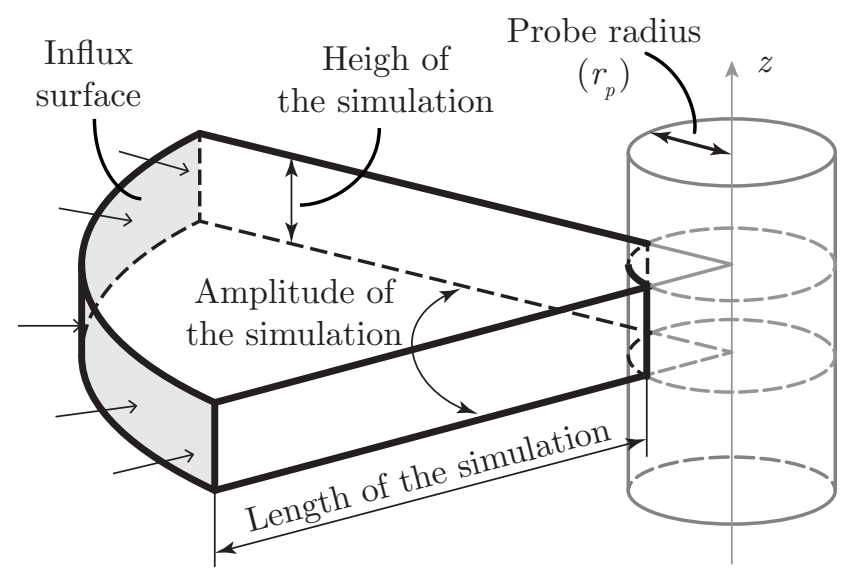

Figure 2. Domain of the developed PIC simulation

A common problem when simulating the contact of a plasma with a surface is the appearance of a source sheath. In our simulation we have avoided this problem by using a particle source for ions which is self-consistently adjusted in order to have a negligible electric field at the influx surface. Ions are assumed to have a Maxwellian distribution in the plasma, but along the presheath they are accelerated by the small electric field present in that zone. For that reason in the presheath ions should be described by a Maxwellian distribution function with a drift velocity in the radial direction.

$$
\begin{aligned}
f_{i}(\vec{v}) & =n_{i 0}\left(\sqrt{\frac{m_{i}}{2 \pi k_{B} T_{i}}}\right)^{3} \\
& \times \exp \left(-\frac{m_{i}}{2 k_{B} T_{i}}\left(\left(v_{r}-v_{d}\right)^{2}+v_{\varphi}^{2}+v_{z}^{2}\right)\right)
\end{aligned}
$$

where $n_{i 0}$ is the ion density at the plasma, $m_{i}$ the ion mass, $T_{i}$ the ion temperature, $k_{B}$ the Boltzmann constant, $v_{r}, v_{\varphi}$ and $v_{z}$ the radial, azimuthal and axial components of the velocity and $v_{d}$ the drift velocity. The ion distribution function shown in Equation (1) is used at the influx surface, and the drift velocity is selfconsistently adjusted in order to fulfil the quasineutral solution there.

Because of the huge computational resources required in particle models, the code of the simulation 
has been parallelised with GPGPU techniques (General Purpose Graphics Processing Units) for the sake of performance. In order to do that, the $\mathrm{CUDA}^{\circledR}$ framework developed by Nvidia has been chosen. Also, to reduce the computational time some algorithmic simplifications have been implemented into the code. As we are interested in high negative biasing potentials of the probe, electrons are completely repelled by the probe, and their density distribution can be described by the following Boltzmann factor:

$$
n_{e}(r)=n_{e 0} \exp \left(\frac{e \phi(r)}{k_{B} T_{e}}\right)
$$

Knowing that, instead of describing electrons in the simulation as particles, we can use the expression in Equation (2) to evaluate their density. Not having to describe the motion of electrons allows us to use a larger time-step, suitable for the, much slower, time scale of ions. Thanks to the previously described considerations, our simulation has achieved great performance even in modest hardware.

\section{Results}

We have used the simulation described in the previous section in order to study the transition from ABR to OML behaviours in a Helium plasma. We have chosen Helium plasmas because it is the gas with which the authors have observed experimentally such a transition. The main benefit of using a computer experiment to study such a transition is that, contrary to what happens in real experiments, we can study the influence of isolated parameters. In our case, we have studied the influence of the dimensionless probe radius for different ion to electron temperature ratios.

To perform the aforementioned study we have used the Sonin-Plot representation [22]. The Sonin-Plot is a dimensionless representation that highlights the difference between the radial and orbital theories, allowing us to know how ions behave in every case. The $y_{\text {sonin }}$ coordinate is the dimensionless ion current at a certain negative probe potential, so-called working point, given by the ratio of the measured ion current to the ion current at plasma potential. Otherwise, the $x_{\text {sonin }}$ coordinate is defined so that it does not depend on the electron number density, $n_{e 0}$, and therefore this quantity can be obtained experimentally by means of cross-plotting in the Sonin-Plot. Both coordinates of the Sonin-Plot are defined in the following equations, and can be written in terms of dimensionless magnitudes as:

$$
\begin{gathered}
y_{\text {sonin }}=\frac{i}{e r_{p} n_{e 0}} \sqrt{\frac{m_{i}}{2 \pi k_{B} T_{e}}}=\frac{I \sqrt{\gamma}}{\sqrt{2 \pi} N_{e 0}} \frac{1}{R_{p}} \\
x_{\text {sonin }}=\frac{i e r_{p}}{\varepsilon_{0}} \sqrt{\frac{m_{i}}{2 \pi k_{B}^{3} T_{e}^{3}}}=\frac{I \sqrt{\gamma}}{\sqrt{2 \pi} N_{e 0}} R_{p}
\end{gathered}
$$

where $\gamma=m_{i} / m_{e}, N_{e 0}=n_{e 0} \lambda_{D}^{3}$ and $I=i \lambda_{D} / e \omega_{p e}$. Also, $i$ being the ion current collected by the probe per unit length, $e$ the elementary charge and $\omega_{p e}$ the electron plasma frequency.

Even though in Figure 1 an almost constant value of the current is observed in the ion saturation zone, since the ion current slightly depends on the biasing potential of the probe, and so the Sonin-Plot does. For this reason, all the results shown in this communication have been obtained for a fixed biasing potential of the probe, $i$. e. working point, $\phi_{p}=-25 k_{B} T_{e} / e$.

As we can see, with the definition given in Equations (3), we can write:

$$
y_{\text {sonin }}=x_{\text {sonin }} / R_{p}^{2}
$$

So, for a fixed dimensionless probe radius, $R_{p}$, the curves $y_{\text {sonin }}\left(x_{\text {sonin }}\right)$ are straight lines that go through the origin of coordinates and whose slope is $1 / R_{p}^{2}$. This can be seen in Figure 3 , where Equation (4) is plotted (thin solid lines) for $R_{p}=0.5,1,2,4$.

In Figure 3 are also shown the predictions of both ABR and the OML theories. On the one hand, the ABR curve is obtained by solving the model numerically, with the RK4 method, for different dimensionless probe radii. Obviously, in the ABR model the value of the ion temperature is zero, so $\beta=0$. On the other hand, the OML model can be solved analytically, so the corresponding curve is given by:

$$
\begin{gathered}
y_{\text {sonin-OML }}=\frac{2}{\sqrt{\pi}} \sqrt{\beta+\frac{\phi_{p} e}{k_{B} T_{e}}} \\
x_{\text {sonin-OML }}=y_{\text {sonin-OML }} R_{p}^{2}
\end{gathered}
$$

With Equations (5), once the biasing potential of the probe is fixed, we can obtain the dependence of the Sonin-Plot in the OML theory on the parameters $\beta$ and $R_{p}$. The OML curve shown in Figure 3 is obtained varying $R_{p}$ for a fixed value $\beta=0$, this is done for the sake of consistency with the ABR curve. Nevertheless, by analysing the Equation (5a), we can see that the dependence on $\beta$ is negligible for $\beta \in[0,1]$ and $\phi_{p}=-25 k_{B} T_{e} / e$.

Finally, the results obtained with the developed simulation are also show in Figure 3 First, as we can see in Figure 3 independently of the probe radius, the ABR model is the theory that properly describes the behaviour of ions in the limiting case $\beta \rightarrow 0$, while for $\beta \neq 0$ the behaviour of ions depends on the probe radius. In order to analyse those results, two regimes have to be defined: small probe radii $\left(R_{p} \lesssim 2\right)$ and large probe radii $\left(R_{p} \gtrsim 2\right)$.

On the one hand, for probe radii approximately smaller than 2 , we can see that as we increase $\beta$, a transition is found from the ABR to the OML theory. We can also see that, the smaller the probe radius the more pronounced the transition.

On the other hand, for probe radii approximately larger than 2 , we can see that as we increase $\beta$, the behaviour of ions can no longer be described by the ABR theory. The difference with the previous case is that the dimensionless current collected by the probe, 


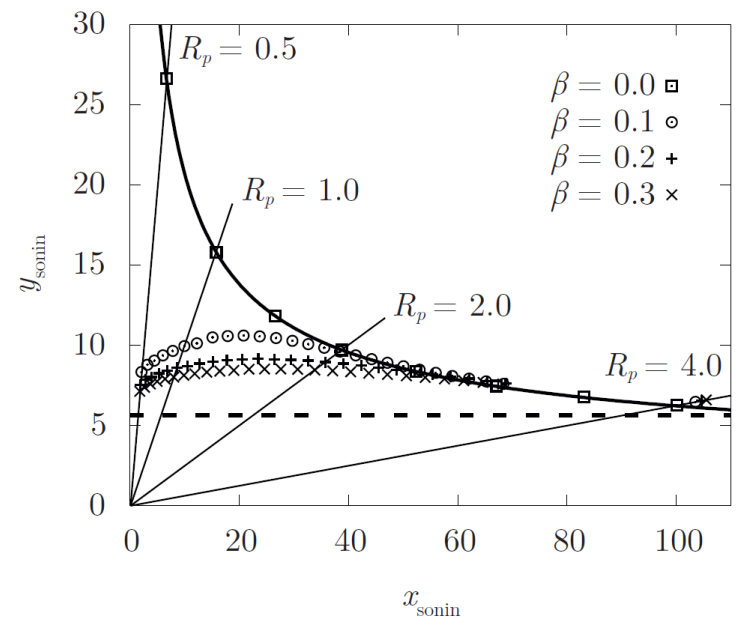

Figure 3. Sonin-Plot representation showing how the behaviour of ions depends on $R_{p}$ for fixed $\beta$ values. Also, ABR (thick solid line), OML (thick dashed line) and constant $R_{p}$ (thin solid) curves are shown.

$I$, does not decrease, as an orbital behaviour would suggest. Contrary to that, the current collected becomes larger than the prediction of the ABR theory. A current larger than the one obtained with the ABR model can be obtained with a radial theory that considers the thermal motion of ions, such as the model developed by Fernández Palop et. al. [13. So, we can conclude that for large probe radii the behaviour of ions remains mostly radial as $\beta$ is increased.

\section{Conclusions}

The influence of the ion to electron temperature ratio, $\beta$, and the dimensionless probe radius, $R_{p}$, on the transition from ABR to OML theories has been studied by means of computer experiments. Two distinctive regimes have been found, one for small probe radii $\left(R_{p} \lesssim 2\right)$ and another for large probe radii $\left(R_{p} \gtrsim 2\right)$. In the small probe radii regime, as the ion to electron temperature ratio is increased, the behaviour of ions goes from ABR to OML. This transition is found to be more pronounced as the dimensionless probe radius is decreased. On the other hand, for large probe radii, as the ion to electron temperature ratio is increased the behaviour of ions departs from the ABR description but does not move towards the OML theory. What happens is that the ion current collected by the probe goes higher than the prediction of the ABR model. For that reason, the behaviour of ions is better described by a radial model that takes into account the ion thermal motion, as the one developed by Fernández Palop et. al. [13].

The use of computer experiments in order to study the behaviour of ions in the surroundings of a cylindrical Langmuir probe has turned out to be very useful. For this reason, further improvements will be implemented into the code to study more complex and realistic conditions, e. g. the influence of collisions in the transition.

\section{Acknowledgements}

The authors greatly acknowledge the support of the FPU Program of the Spanish Ministry of Education (ref. AP2010/0639) and the Spanish Ministry of Science and Innovation (ref. No FIS2010-19951) which is partially financed with FEDER funds.

\section{References}

[1] T. E. Sheridan. Ion focusing by an expanding, two-dimensional plasma sheath. Applied Physics Letters, 68(14):1918-1920, 1996.

[2] Shi Qian, Huiliang Cao, Xuanyong Liu, and Chuanxian Ding. Nanotube array controlled carbon plasma deposition. Applied Physics Letters, 102(24):243109, jun 2013.

[3] G. D. Severn, Xu Wang, Eunsuk Ko, and N. Hershkowitz. Experimental Studies of the Bohm Criterion in a Two-Ion-Species Plasma Using Laser-Induced Fluorescence. Physical Review Letters, 90(14):145001, apr 2003.

[4] V. Demidov, C. DeJoseph, and A. Kudryavtsev. Anomalously High Near-Wall Sheath Potential Drop in a Plasma with Nonlocal Fast Electrons. Physical Review Letters, 95(21):215002, nov 2005.

[5] Dongsoo Lee, Lutfi Oksuz, and Noah Hershkowitz. Exact Solution for the Generalized Bohm Criterion in a Two-Ion-Species Plasma. Physical Review Letters, 99(15):155004, oct 2007.

[6] M. D. Campanell, A. V. Khrabrov, and I. D. Kaganovich. Absence of Debye Sheaths due to Secondary Electron Emission. Physical Review Letters, 108(25):255001, jun 2012.

[7] J.I. Fernández Palop, V. Colomer, J. Ballesteros, M.A. Hernández, and A. Dengra. Theoretical aspects of the metal-electronegative plasma interface. Surface and Coatings Technology, 84(1-3):341-347, oct 1996.

[8] H. M. Mott-Smith and Irving Langmuir. The Theory of Collectors in Gaseous Discharges. Physical Review, 28(4):727-763, oct 1926.

[9] Ira B. I.B. Bernstein and I.N. Irving N. Rabinowitz. Theory of Electrostatic Probes in a Low-Density Plasma. Physics of Fluids, 2(2):112-121, 1959.

[10] James G Laframboise. Theory of spherical and cylindrical langmuir probes in a collisionless, maxwellian plasma at rest. University of Toronto Institute for Aerospace Studies, (Report - 100):1-216, 1966. Unpublished.

[11] J E Allen, R L F Boyd, and P Reynolds. The Collection of Positive Ions by a Probe Immersed in a Plasma. Proceedings of the Physical Society. Section B, 70(3):297-304, mar 1957.

[12] F F Chen. Numerical computations for ion probe characteristics in a collisionless plasma. Journal of Nuclear Energy. Part C, Plasma Physics, Accelerators, Thermonuclear Research, 7(1):47-67, jan 1965.

[13] J. I. Fernández Palop, J. Ballesteros, V. Colomer, and M. A. Hernández. Theoretical ion current to cylindrical Langmuir probes for finite ion temperature values. Journal of Physics D: Applied Physics, 29(11):2832-2840, nov 1996. 
[14] R. Morales Crespo, J. I. Fernández Palop, M. A. Hernández, and J. Ballesteros. Analytical fit of the I-V probe characteristic for finite ion temperature values: Justification of the radial model applicability. Journal of Applied Physics, 95(6):2982, 2004.

[15] F F Chen. Langmuir probes in RF plasma: surprising validity of OML theory. Plasma Sources Science and Technology, 18(3):035012, aug 2009.

[16] M. a. Hassouba, a. R. Galaly, and U. M. Rashed. Analysis of cylindrical Langmuir probe using experiment and different theories. Plasma Physics Reports, 39(3):255-262, mar 2013.

[17] E Passoth, P Kudrna, C Csambal, J F Behnke, M Tichý, and V Helbig. An experimental study of plasma density determination by a cylindrical Langmuir probe at different pressures and magnetic fields in a cylindrical magnetron discharge in heavy rare gases. Journal of Physics D: Applied Physics, 30(12):1763-1777, jun 1997.

[18] J. M. Díaz-Cabrera, M. V. Lucena-Polonio, J. I. Fernández Palop, R. Morales Crespo, M. A. Hernández, A. Tejero-del Caz, and J. Ballesteros. Experimental study of the ion current to a cylindrical Langmuir probe taking into account a finite ion temperature. Journal of Applied Physics, 111(6):063303, 2012.

[19] F F Chen, J D Evans, and Wade Zawalski.

Calibration of Langmuir probes against microwaves and plasma oscillation probes. Plasma Sources Science and Technology, 21(5):055002, oct 2012.

[20] J. M. Díaz-Cabrera, J. Ballesteros, J I Fernández Palop, and A. Tejero-del Caz. Experimental radial motion to orbital motion transition in cylindrical Langmuir probes in low pressure plasmas. Plasma Sources Science and Technology, 24(2):025026, 2015.

[21] R W Hockney and J W Eastwood. Computer Simulation Using Particles. IOP Publishing Ltd., 1988.

[22] A. A. Sonin. Free-molecule Langmuir probe and its use in flow-field studies. AIAA Journal, 4(9):1588-1596, sep 1966. 